\title{
C. T. C. Wall's contributions to the topology of manifolds
}

\author{
Sylvain Cappell, ${ }^{*}$ Andrew Ranicki, ${ }^{\dagger}$ \\ and Jonathan Rosenberg ${ }^{\ddagger}$
}

Note. Numbered references in this survey refer to the Research Papers in Wall's Publication List.

\section{A quick overview}

C. T. C. Wall ${ }^{1}$ spent the first half of his career, roughly from 1959 to 1977, working in topology and related areas of algebra. In this period, he produced more than 90 research papers and two books, covering

- cobordism groups,

- the Steenrod algebra,

- homological algebra,

- manifolds of dimensions $3,4, \geq 5$,

- quadratic forms,

- finiteness obstructions,

- embeddings,

- bundles,

- Poincaré complexes,

- surgery obstruction theory,

* Partially supported by NSF Grant \# DMS-96-26817.

$\dagger$ This is an expanded version of a lecture given at the Liverpool Singularities Meeting in honor of Wall in August 1996.

$¥$ Partially supported by NSF Grant \# DMS-96-25336.

${ }^{1}$ Charles Terence Clegg Wall, known in his papers by his initials C. T. C. and to his friends as Terry. 
- homology of groups,

- 2-dimensional complexes,

- the topological space form problem,

- computations of $K$ - and $L$-groups,

- and more.

One quick measure of Wall's influence is that there are two headings in the Mathematics Subject Classification that bear his name:

- 57Q12 (Wall finiteness obstruction for $C W$ complexes).

- 57R67 (Surgery obstructions, Wall groups).

Above all, Wall was responsible for major advances in the topology of manifolds. Our aim in this survey is to give an overview of how his work has advanced our understanding of classification methods. Wall's approaches to manifold theory may conveniently be divided into three phases, according to the scheme:

1. All manifolds at once, up to cobordism (1959-1961).

2. One manifold at a time, up to diffeomorphism (1962-1966).

3. All manifolds within a homotopy type (1967-1977).

\section{Cobordism}

Two closed $n$-dimensional manifolds $M_{1}^{n}$ and $M_{2}^{n}$ are called cobordant if there is a compact manifold with boundary, say $W^{n+1}$, whose boundary is the disjoint union of $M_{1}$ and $M_{2}$. Cobordism classes can be added via the disjoint union of manifolds, and multiplied via the Cartesian product of manifolds. Thom (early 1950's) computed the cobordism ring $\mathfrak{N}_{*}$ of unoriented smooth manifolds, and began the calculation of the cobordism ring $\Omega_{*}$ of oriented smooth manifolds.

After Milnor showed in the late 1950's that $\Omega_{*}$ contains no odd torsion, Wall $[1,3]$ completed the calculation of $\Omega_{*}$. This was the ultimate achievement of the pioneering phase of cobordism theory. One version of Wall's main result is easy to state:

Theorem 2.1 (Wall [3]) All torsion in $\Omega_{*}$ is of order 2. The oriented cobordism class of an oriented closed manifold is determined by its StiefelWhitney and Pontrjagin numbers.

For a fairly detailed discussion of Wall's method of proof and of its remarkable corollaries, see [Ros]. 
Wall's contributions to the topology of manifolds

\section{Structure of manifolds}

What is the internal structure of a cobordism? Morse theory has as one of its main consequences (as pointed out by Milnor) that any cobordism between smooth manifolds can be built out of a sequence of handle attachments.

Definition 3.1 Given an $m$-dimensional manifold $M$ and an embedding $S^{r} \times D^{m-r} \hookrightarrow M$, there is an associated elementary cobordism $(W ; M, N)$ obtained by attaching an $(r+1)$-handle to $M \times I$. The cobordism $W$ is the union

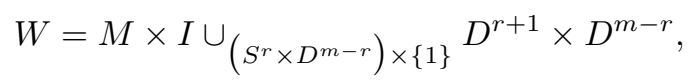

and $N$ is obtained from $M$ by deleting $S^{r} \times D^{m-r}$ and gluing in $S^{r} \times D^{m-r}$ in its place:

$$
N=\left(M \backslash\left(S^{r} \times D^{m-r}\right)\right) \cup_{S^{r} \times S^{m-r-1}} D^{r+1} \times S^{m-r-1} .
$$

The process of constructing $N$ from $M$ is called surgery on an $r$-sphere, or surgery in dimension $r$ or in codimension $m-r$. Here $r=-1$ is allowed, and amounts to letting $N$ be the disjoint union of $M$ and $S^{m}$.

Any cobordism may be decomposed into such elementary cobordisms. In particular, any closed smooth manifold may be viewed as a cobordism between empty manifolds, and may thus be decomposed into handles.

Definition 3.2 A cobordism $W^{n+1}$ between manifolds $M^{n}$ and $N^{n}$ is called an $h$-cobordism if the inclusions $M \hookrightarrow W$ and $N \hookrightarrow W$ are homotopy equivalences.

The importance of this notion stems from the $h$-cobordism theorem of Smale (ca. 1960), which showed that if $M$ and $N$ are simply connected and of dimension $\geq 5$, then every $h$-cobordism between $M$ and $N$ is a cylinder $M \times I$. The crux of the proof involves handle cancellations as well as Whitney's trick for removing double points of immersions in dimension $>4$. In particular, if $M^{n}$ and $N^{n}$ are simply connected and $h$-cobordant, and if $n>4$, then $M$ and $N$ are diffeomorphic (or $P L$-homeomorphic, depending on whether one is working in the smooth or the $P L$ category).

For manifolds which are not simply connected, the situation is more complicated and involves the fundamental group. But Smale's theorem was extended a few years later by Barden, ${ }^{2}$ Mazur, and Stallings to give the $s$ cobordism theorem, which (under the same dimension restrictions) showed that the possible $h$-cobordisms between $M$ and $N$ are in natural bijection with the elements of the Whitehead group Wh $\pi_{1}(M)$. The bijection sends

\footnotetext{
${ }^{2}$ One of Wall's students!
} 
an $h$-cobordism $W$ to the Whitehead torsion of the associated homotopy equivalence from $M$ to $W$, an invariant from algebraic $K$-theory that arises from the combinatorics of handle rearrangements. One consequence of this is that if $M$ and $N$ are $h$-cobordant and the Whitehead torsion of the $h$ cobordism vanishes (and in particular, if $\mathrm{Wh} \pi_{1}(M)=0$, which is the case for many $\pi_{1}$ 's of practical interest), then $M$ and $N$ are again diffeomorphic (assuming $n>4$ ).

The use of the Whitney trick and the analysis of handle rearrangements, crucial to the proof of the $h$-cobordism and $s$-cobordism theorems, became the foundation of Wall's work on manifold classification.

\section{4-Manifolds}

Milnor, following J. H. C. Whitehead, observed in 1956 that a simply connected 4-dimensional manifold $M$ is classified up to homotopy equivalence by its intersection form, the non-degenerate symmetric bilinear from on $\mathrm{H}_{2}(M ; \mathbb{Z})$ given by intersection of cycles, or in the dual picture, by the cup-product

$$
H^{2}(M ; \mathbb{Z}) \times H^{2}(M ; \mathbb{Z}) \rightarrow H^{4}(M ; \mathbb{Z}) \stackrel{\cong}{\longrightarrow} \mathbb{Z}
$$

Note that the isomorphism $H^{4}(M ; \mathbb{Z}) \rightarrow \mathbb{Z}$, and thus the form, depends on the orientation.

Classification of 4-dimensional manifolds up to homeomorphism or diffeomorphism, however, has remained to this day one of the hardest problems in topology, because of the failure of the Whitney trick in this dimension. Wall succeeded in 1964 to get around this difficulty at the expense of "stabilizing." He used handlebody theory to obtain a stabilized version of the $h$-cobordism theorem for 4-dimensional manifolds:

Theorem 4.1 (Wall [19]) For two simply connected smooth closed oriented 4-manifolds $M_{1}$ and $M_{2}$, the following are equivalent:

1. they are h-cobordant;

2. they are homotopy equivalent (in a way preserving orientation);

3. they have the same intersection form on middle homology.

If these conditions hold, then $M_{1} \# k\left(S^{2} \times S^{2}\right)$ and $M_{2} \# k\left(S^{2} \times S^{2}\right)$ are diffeomorphic (in a way preserving orientation) for $k$ sufficiently large (depending on $M_{1}$ and $\left.M_{2}\right)$.

Note incidentally that the converse of the above theorem is not quite true: $M_{1} \# k\left(S^{2} \times S^{2}\right)$ and $M_{2} \# k\left(S^{2} \times S^{2}\right)$ are diffeomorphic (in a way 
preserving orientation) for $k$ sufficiently large if and only if the intersection forms of $M_{1}$ and $M_{2}$ are stably isomorphic (where stability refers to addition of the hyperbolic form $\left(\begin{array}{ll}0 & 1 \\ 1 & 0\end{array}\right)$ ).

From the 1960's until Donaldson's work in the 1980's, Theorem 4.1 was basically the only significant result on the diffeomorphism classification of simply-connected 4-dimensional manifolds. Thanks to Donaldson's work, we now know that the stabilization in the theorem (with respect to addition of copies of $S^{2} \times S^{2}$ ) is unavoidable, in that without it, nothing like Theorem 4.1 could be true.

\section{$5 \quad$ Highly connected manifolds}

The investigation of simply connected 4-dimensional manifolds suggested the more general problem of classifying $(n-1)$-connected $2 n$-dimensional manifolds, for all $n$. The intersection form on middle homology again appears as a fundamental algebraic invariant of oriented homotopy type. In fact this invariant also makes sense for an $(n-1)$-connected $2 n$-dimensional manifold $M$ with boundary a homology sphere $\partial M=\Sigma^{2 n-1}$. If $\partial M$ is a homotopy sphere, it has a potentially exotic differentiable structure for $n \geq 4 .^{3}$

Theorem 5.1 (Wall [10]) For $n \geq 3$ the diffeomorphism classes of differentiable $(n-1)$-connected $2 n$-dimensional manifolds with boundary a homotopy sphere are in natural bijection with the isomorphism classes of $\mathbb{Z}$-valued non-degenerate $(-1)^{n}$-symmetric forms with a quadratic refinement in $\pi_{n}(B S O(n))$.

(The form associated to a manifold $M$ is of course the intersection form on the middle homology $H_{n}(M ; \mathbb{Z})$. This group is isomorphic to $\pi_{n}(M)$, by the Hurewicz theorem, so every element is represented by a map $S^{n} \rightarrow M^{2 n}$. By the Whitney trick, this can be deformed to an embedding, with normal bundle classified by an element of $\pi_{n}(B S O(n))$. The quadratic refinement is defined by this homotopy class.)

The sequence of papers $[15,16,22,23,37,42]$ extended this diffeomorphism classification to other types of highly-connected manifolds, using a combination of homotopy theory and the algebra of quadratic forms. These papers showed how far one could go in the classification of manifolds without surgery theory.

\footnotetext{
${ }^{3}$ It was the study of the classification of 3-connected 8-dimensional manifolds with boundary which led Milnor to discover the existence of exotic spheres in the first place. [Mil]
} 


\section{Finiteness obstruction}

Recall that if $X$ is a space and $f: S^{r} \rightarrow X$ is a map, the space obtained from $X$ by attaching an $(r+1)$-cell is $X \cup_{f} D^{r+1}$. A $C W$ complex is a space obtained from $\emptyset$ by attaching cells. It is called finite if only finitely many cells are used. One of the most natural questions in topology is:

When is a space homotopy equivalent to a finite $C W$ complex?

A space $X$ is called finitely dominated if it is a homotopy retract of a finite $C W$ complex $K$, i.e., if there exist maps $f: X \rightarrow K, g: K \rightarrow X$ and a homotopy $g f \simeq 1: X \rightarrow X$. This is clearly a necessary condition for $X$ to be of the homotopy type of a finite $C W$ complex. Furthermore, for spaces of geometric interest, finite domination is much easier to verify than finiteness. For example, already in 1932 Borsuk had proved that every compact $A N R$, such as a compact topological manifold, is finitely dominated. So another question arises:

Is a finitely dominated space homotopy equivalent to a finite $C W$ complex?

This question also has roots in the study of the free actions of finite groups on spheres. A group with such an action necessarily has periodic cohomology. In the early 1960's Swan had proved that a finite group $\pi$ with cohomology of period $q$ acts freely on an infinite $C W$ complex $Y$ homotopy equivalent to $S^{q-1}$, with $Y / \pi$ finitely dominated, and that $\pi$ acts freely on a finite complex homotopy equivalent to $S^{q-1}$ if and only if an algebraic $K$-theory invariant vanishes. Swan's theorem was in fact a special case of the following general result.

Theorem 6.1 (Wall $[26,43])$ A finitely dominated space $X$ has an associated obstruction $[X] \in \widetilde{K}_{0}\left(\mathbb{Z}\left[\pi_{1}(X)\right]\right)$. The space $X$ is homotopy equivalent to a finite $C W$ complex if and only if this obstruction vanishes.

The obstruction defined in this theorem, now universally called the Wall finiteness obstruction, is a fundamental algebraic invariant of non-compact topology. It arises as follows. If $K$ is a finite $C W$ complex dominating $X$, then the cellular chain complex of $K$, with local coefficients in the group $\operatorname{ring} \mathbb{Z}\left[\pi_{1}(X)\right]$, is a finite complex of finitely generated free modules. The domination of $X$ by $K$ thus determines a direct summand subcomplex of a finite chain complex, attached to $X$. Since a direct summand in a free module is projective, this chain complex attached to $X$ consists of finitely generated projective modules. The Wall obstruction is a kind of "Euler characteristic" measuring whether or not this chain complex is chain equivalent to a finite complex of finitely generated free modules. 
The Wall finiteness obstruction has turned out to have many applications to the topology of manifolds, most notably the Siebenmann end obstruction for closing tame ends of open manifolds.

\section{Surgery theory and the Wall groups}

The most significant of all of Wall's contributions to topology was undoubtedly his development of the general theory of non-simply-connected surgery. As defined above, surgery can be viewed as a means of creating new manifolds out of old ones. One measure of Wall's great influence was that when other workers (too numerous to list here) made use of surgery, they almost invariably drew upon Wall's contributions.

As a methodology for classifying manifolds, surgery was first developed in the 1961 work of Kervaire and Milnor [KM] classifying homotopy spheres in dimensions $n \geq 6$, up to $h$-cobordism (and hence, by Smale's theorem, up to diffeomorphism). If $W^{n}$ is a parallelizable manifold with homotopy sphere boundary $\partial W=\Sigma^{n-1}$, then it is possible to kill the homotopy groups of $W$ by surgeries if and only if an obstruction

$$
\sigma(W) \in P_{n}= \begin{cases}\mathbb{Z} & \text { if } n \equiv 0(\bmod 4), \\ \mathbb{Z} / 2 & \text { if } n \equiv 2(\bmod 4), \\ 0 & \text { if } n \text { is odd }\end{cases}
$$

vanishes. Here

$$
\sigma(W)= \begin{cases}\operatorname{signature}(W) \in \mathbb{Z} & \text { if } n \equiv 0(\bmod 4), \\ \operatorname{Arf} \operatorname{invariant}(W) \in \mathbb{Z} / 2 & \text { if } n \equiv 2(\bmod 4)\end{cases}
$$

In 1962 Browder [Br] used the surgery method to prove that, for $n \geq 5$, a simply-connected finite $C W$ complex $X$ with $n$-dimensional Poincaré duality

$$
H^{n-*}(X) \cong H_{*}(X)
$$

is homotopy equivalent to a closed $n$-dimensional differentiable manifold if and only if there exists a vector bundle $\eta$ with spherical Thom class such that an associated invariant $\sigma \in P_{n}$ (the simply connected surgery obstruction) is 0 . The result was proved by applying Thom transversality to $\eta$ to obtain a suitable degree-one map $M \rightarrow X$ from a manifold, and then killing the kernel of the induced map on homology. For $n=4 k$ the invariant $\sigma \in P_{4 k}=\mathbb{Z}$ is one eighth of the difference between signature $(X)$ and the $4 k$-dimensional component of the $\mathcal{L}$-genus of $-\eta$. (The minus sign comes from the fact that the tangent and normal bundles are stably the negatives of one another.) In this case, the result is a converse of the Hirzebruch signature theorem. In other words, $X$ is homotopy-equivalent to a differentiable manifold if and only if the formula of the theorem holds 
with $\eta$ playing the role of the stable normal bundle. The hardest step was to find enough embedded spheres with trivial normal bundle in the middle dimension, using the Whitney embedding theorem for embeddings $S^{m} \subset M^{2 m}$ - this requires $\pi_{1}(M)=\{1\}$ and $m \geq 3$. Also in 1962, Novikov initiated the use of surgery in the study of the uniqueness of differentiable manifold structures in the homotopy type of a manifold, in the simplyconnected case.

From about 1965 until 1970, Wall developed a comprehensive surgery obstruction theory, which also dealt with the non-simply-connected case. The extension to the non-simply-connected case involved many innovations, starting with the correct generalization of the notion of Poincaré duality. A connected finite $C W$ complex $X$ is called a Poincaré complex [44] of dimension $n$ if there exists a fundamental homology class $[X] \in H_{n}(X ; \mathbb{Z})$ such that cap product with $[X]$ induces isomorphisms from cohomology to homology with local coefficients,

$$
H^{n-*}\left(X ; \mathbb{Z}\left[\pi_{1}(X)\right]\right) \stackrel{\cong}{\longrightarrow} H_{*}\left(X ; \mathbb{Z}\left[\pi_{1}(X)\right]\right) .
$$

This is obviously a necessary condition for $X$ to have the homotopy type of a closed $n$-dimensional manifold. A normal map or surgery problem

$$
(f, b): M^{n} \rightarrow X
$$

is a degree-one map $f: M \rightarrow X$ from a closed $n$-dimensional manifold to an $n$-dimensional Poincaré complex $X$, together with a bundle map $b: \nu_{M} \rightarrow \eta$ so that

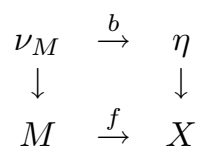

commutes.

Wall defined ([41], [W1]) the surgery obstruction groups $L_{*}(A)$ for any ring with involution $A$, using quadratic forms over $A$ and their automorphisms. They are more elaborate versions of the Witt groups of fields studied by algebraists.

Theorem 7.1 (Wall, [41], [W1]) A normal map $(f, b): M \rightarrow X$ has a surgery obstruction

$$
\sigma_{*}(f, b) \in L_{n}\left(\mathbb{Z}\left[\pi_{1}(X)\right]\right),
$$

and $(f, b)$ is normally bordant to a homotopy equivalence if (and for $n \geq 5$ only if) $\sigma_{*}(f, b)=0$.

One of Wall's accomplishments in this theorem, quite new at the time, was to find a way to treat both even-dimensional and odd-dimensional manifolds in the same general framework. Another important accomplishment 
was the recognition that surgery obstructions live in groups depending on the fundamental group, but not on any other aspect of $X$ (except for the dimension modulo 4 and the orientation character $w_{1}$. Here we have concentrated on the oriented case, $\left.w_{1}=0\right)$. In general, the groups $L_{n}\left(\mathbb{Z}\left[\pi_{1}(X)\right]\right)$ are not so easy to compute (more about this below and elsewhere in this volume!), but in the simply-connected case, $\pi_{1}(X)=\{1\}$, they are just the Kervaire-Milnor groups, $L_{n}(\mathbb{Z}[\{1\}])=P_{n}$.

Wall formulated various relative version of Theorem 7.1 for manifolds with boundary, and manifold $n$-ads. An important special case is often quoted, which formalizes the idea that the "surgery obstruction groups only depend on the fundamental group." This is the celebrated:

Theorem 7.2 " $\pi-\pi$ Theorem" ([W1], 3.3) Suppose one is given a surgery problem $(f, b): M^{n} \rightarrow X$, where $M$ and $X$ each have connected non-empty boundary, and suppose $\pi_{1}(\partial X) \rightarrow \pi_{1}(X)$ is an isomorphism. Also assume that $n \geq 6$. Then $(f, b)$ is normally cobordant to a homotopy equivalence of pairs.

The most important consequence of Wall's theory, which appeared for the first time in Chapter 10 of [W1], is that it provides a "classification" of the manifolds in a fixed homotopy type (in dimensions $\geq 5$ in the absolute case, $\geq 6$ in the relative case). The formulation, in terms of the "surgery exact sequence," was based on the earlier work of Browder, Novikov, and Sullivan in the simply connected case. The basic object of study is the structure set $\mathcal{S}(X)$ of a Poincaré complex $X$. This is the set of all homotopy equivalences (or perhaps simple homotopy equivalences, depending on the way one wants to formulate the theory) $M \stackrel{f}{\longrightarrow} X$, where $M$ is a manifold, modulo a certain equivalence relation: $M \stackrel{f}{\longrightarrow} X$ and $M^{\prime} \stackrel{f^{\prime}}{\longrightarrow} X$ are considered equivalent if there is a diffeomorphism $\phi: M \rightarrow M^{\prime}$ such that $f^{\prime} \circ \phi$ is homotopic to $f$. One should think of $\mathcal{S}(X)$ as "classifying all manifold structures on the homotopy type of $X$."

Theorem 7.3 (Wall [W1], Theorem 10.3 et seq.) Under the above dimension restrictions, the structure set $\mathcal{S}(X)$ of a Poincaré complex $X$ is non-empty if and only if there exists a normal map $(f, b): M \rightarrow X$ with surgery obstruction $\sigma_{*}(f, b)=0 \in L_{n}\left(\mathbb{Z}\left[\pi_{1}(X)\right]\right)$. If non-empty, $\mathcal{S}(X)$ fits into an exact sequence (of sets)

$$
L_{n+1}\left(\mathbb{Z}\left[\pi_{1}(X)\right]\right) \rightarrow \mathcal{S}(X) \rightarrow \mathcal{T}(X) \rightarrow L_{n}\left(\mathbb{Z}\left[\pi_{1}(X)\right]\right),
$$

where $\mathcal{T}(X)=[X, G / O]$ classifies "tangential data."

Much of [W1] and many of Wall's papers in the late 1960's and early 1970 's were taken up with calculations and applications. We mention only 
a few of the applications: a new proof of the theorem of Kervaire characterizing the fundamental groups of high-dimensional knot complements, results on realization of Poincaré (i.e., homotopy-theoretic) embeddings in manifolds by actual embeddings of submanifolds, classification of free actions of various types of discrete groups on manifolds (for example, free involutions on spheres), the classification of "fake projective spaces," "fake lens spaces," "fake tori," and more. The work on the topological space form problem (free actions on spheres) is particularly significant: the $C W$ complex version had already motivated the work of Swan and Wall on the finiteness obstruction discussed above, while the manifold version was one of the impulses for Wall's (and others') extensive calculations of the $L$-groups of finite groups.

\section{PL and topological manifolds}

While surgery theory was originally developed in the context of smooth manifolds, it was soon realized that it works equally well in the $P L$ category of combinatorial manifolds. Indeed, the book [W1] was written in the language of $P L$ manifolds. Wall's theory has the same form in both categories, and in fact the surgery obstruction groups are the same, regardless of whether one works in the smooth or in the $P L$ category. The only differences are that for the $P L$ case, vector bundles must be replaced by $P L$ bundles, and in theorem $7.3, G / O$ should be replaced by $G / P L$.

Passage from the $P L$ to the topological category was a much trickier step (even though we now know that $G / P L$ more closely resembles $G / T O P$ than $G / O)$. Wall wrote in the introduction to [44]:

This paper was originally planned when the only known fact about topological manifolds (of dimension $>3$ ) was that they were Poincaré complexes. Novikov's proof of the topological invariance of rational Pontrjagin classes and subsequent work in the same direction has changed this ....

Novikov's work introduced the torus $T^{n}$ as an essential tool in the study of topological manifolds. A fake torus is a manifold which is homotopy equivalent to $T^{n}$. The surgery theoretic classification of PL fake tori in dimensions $\geq 5$ by Wall and by Hsiang and Shaneson [HS] was an essential tool in the work of Kirby $[\mathrm{K}]$ and Kirby-Siebenmann [KS] on the structure theory of topological manifolds. (See also $[\mathrm{KSW}]$.) This in turn made it possible to extend surgery theory to the topological category. 
Wall's contributions to the topology of manifolds

\section{Invariance properties of the signature}

Wall made good use of the signature invariants of quadratic forms and manifolds. We pick out three particular cases:

1. One immediate (but non-trivial) consequence of the Hirzebruch signature theorem is that if $\widetilde{M}$ is a $k$-fold covering of a closed manifold $M$, then

$$
\text { signature }(\widetilde{M})=k \cdot \operatorname{signature}(M) \in \mathbb{Z}
$$

It is natural to ask whether this property is special to manifolds, or whether it holds for Poincaré complexes in general. But in [44], Wall constructed examples of 4-dimensional Poincaré complexes $X$ where this fails, and hence such $X$ are not homotopy equivalent to manifolds.

2. For finite groups $\pi$, Wall [W1] showed that the surgery obstruction groups $L_{*}(\mathbb{Z}[\pi])$ are finitely generated abelian groups, and that the torsion-free part of these groups is determined by a collection of signature invariants called the multisignature. This work led to a series of deep interactions between algebraic number theory and geometric topology.

3. The Novikov additivity property of the signature is that the signature of the boundary-connected union of manifolds is the sum of the signatures. In [54], Wall showed that this additivity fails for unions of manifolds along parts of boundaries which are not components, and obtained a homological expression for the non-additivity of the signature (which is also known as the Maslov index).

\section{Homological and combinatorial group theory}

Wall's work on surgery theory led to him to several problems in combinatorial group theory. One of these was to determine what groups can be the fundamental groups of aspherical Poincaré complexes. Such groups are called Poincaré duality groups. There are evident connections with the topology of manifolds:

([W2], problem G2, p. 391) Is every Poincaré duality group $\Gamma$ the fundamental group of a closed $K(\Gamma, 1)$ manifold? Smooth manifold? Manifold unique up to homeomorphism? (It will not be unique up to diffeomorphism.) 
([W2], problem F16, p. 388) Let $\Gamma$ be a Poincaré duality group of dimension $\geq 3$. Is the 'fundamental group at infinity' of $\Gamma$ necessarily trivial? This is known in many cases, e.g. if $\Gamma$ has a finitely presented normal subgroup $\Gamma^{\prime}$ of infinite index and either $\Gamma^{\prime}$ or $\Gamma / \Gamma^{\prime}$ has one end. In dimensions $\geq 5$ it is equivalent to having the universal cover of a compact $K(\Gamma, 1)$ manifold homeomorphic to euclidean space.

For more on the subsequent history of these problems, see [FRR] and [D].

\section{References}

[Br] W. Browder, Homotopy type of differentiable manifolds, in Proc. Arhus Topology Conference (1962), reprinted in Novikov Conjectures, Index Theorems and Rigidity, Lond. Math. Soc. Lecture Notes 226, Cambridge Univ. Press (1995), 97-100.

[D] M. Davis, Poincaré duality groups, this volume.

[FRR] S. Ferry, A. Ranicki and J. Rosenberg (editors), Novikov Conjectures, Index Theorems and Rigidity, Lond. Math. Soc. Lecture Notes 226, 227, Cambridge Univ. Press (1995).

[HS] W.-C. Hsiang and J. Shaneson, Fake tori, in Topology of Manifolds, Proc. Conf. at Univ. of Georgia, 1969, Markham, Chicago (1970), 18-51.

[KM] M. Kervaire and J. Milnor, Groups of homotopy spheres, I. Ann. of Math. 77 (1963), 504-537.

[K] R. Kirby, Stable homeomorphisms and the annulus conjecture, Ann. of Math. 89 (1969), 575-583.

[KS] R. Kirby and L. Siebenmann, Foundational Essays on Topological Manifolds, Smoothings and Triangulations, Ann. of Math. Studies 88, Princeton Univ. Press, 1977.

[KSW] R. Kirby, L. Siebenmann, and C. T. C. Wall, The annulus conjecture and triangulation, Abstract \# 69T-G27, Notices Amer. Math. Soc. 16 (1969), 432.

[KT] R. C. Kirby and L. R. Taylor, A survey of 4-manifolds through the eyes of surgery, vol. 2 of this collection.

[Mil] J. Milnor, Classification of $(n-1)$-connected $2 n$-dimensional manifolds and the discovery of exotic spheres, this volume. 
[Ros] J. Rosenberg, Reflections on C. T. C. Wall's work on cobordism, vol. 2 of this collection.

[W1] C. T. C. Wall, Surgery on Compact Manifolds, (London Math. Soc. monographs, no. 1), Academic Press, London and New York, 1970. 2nd edition, A.M.S. Surveys and Monographs, A.M.S., 1999.

[W2] C. T. C. Wall (editor), Homological Group Theory, Lond. Math. Soc. Lecture Notes 36, Cambridge Univ. Press (1979).

Author AdDREsses:

S. C.: Courant Institute for the Mathematical Sciences

New York University

215 Mercer St.

New York, NY 10012-1110

USA

email: cappell@cims.nyu.edu

A. R.: Department of Mathematics

University of Edinburgh

The King's Buildings

Edinburgh EH9 3JZ

Scotland, UK

email: aar@maths.ed.ac.uk

J. R.: Department of Mathematics

University of Maryland

College Park, MD 20742-4015

USA

email: jmr@math.umd.edu 


\section{T. C. Wall's publication list}

\section{Books}

A geometric introduction to topology, vi, 168 pp., Addison Wesley, 1971; reprinted in paperback by Dover, 1993.

Surgery on compact manifolds, London Math. Soc. Monographs no. 1, x, 280 pp. Academic Press, 1970; 2nd edition (ed. A.A. Ranicki), Amer. Math. Soc. Surveys and Monographs 69, A.M.S., 1999.

Proceedings of Liverpool Singularities Symposium I., II. (edited), Lecture Notes in Math. 192, 209, Springer, 1971.

Homological group theory (edited), London Math. Soc. Lecture Notes 36, Cambridge University Press, 1979.

The geometry of topological stability (with A.A. du Plessis), viii, 572 pp., London Math. Soc. Monographs, New Series, no. 9, Oxford University Press, 1995.

\section{Research papers}

1. Note on the cobordism ring, Bull. Amer. Math. Soc. 65 (1959) 329331 .

2. On a result in polynomial rings, Proc. Camb. Phil. Soc. 56 (1960) 104-108.

3. Determination of the cobordism ring, Ann. of Math. 72 (1960) 292311.

4. Generators and relations for the Steenrod algebra, Ann. of Math. 72 (1960) 429-444.

5. Rational Euler characteristics, Proc. Camb. Phil. Soc. 57 (1961) $182-183$.

6. Cobordism of pairs, Comm. Math. Helv. 35 (1961) 136-145.

7. Resolutions for extensions of groups, Proc. Camb. Phil. Soc. 57 (1961) 251-255.

8. On the cohomology of certain groups, Proc. Camb. Phil. Soc. 57 (1961) 731-733. 
9. Killing the middle homotopy groups of odd dimensional manifolds, Trans. Amer. Math. Soc. 103 (1962) 421-433.

10. Classification of $(n-1)$-connected $2 n$-manifolds, Ann. of Math. $\mathbf{7 5}$ (1962) 163-189.

11. Cobordism exact sequences for differential and combinatorial manifolds, Ann. of Math. 77 (1963) 1-15.

12. On the orthogonal groups of unimodular quadratic forms, Math. Ann. 147 (1962) 328-338.

13. The action of $\Gamma_{2 n}$ on $(n-1)$-connected $2 n$-manifolds, Proc. Amer. Math. Soc. 13 (1962) 943-944.

14. A characterisation of simple modules over the Steenrod algebra mod. 2, Topology 1 (1962) 249-254.

15. Classification problems in differential topology I. Classification of handlebodies, Topology 2 (1963) 253-261.

16. Classification problems in differential topology II. Diffeomorphisms of handlebodies, Topology 2 (1963) 263-272.

17. On the orthogonal groups of unimodular quadratic forms II., J. reine angew. Math. 213 (1963) 122-136.

18. Diffeomorphisms of 4-manifolds, J. London Math. Soc. 39 (1964) 131-140.

19. On simply-connected 4-manifolds, J. London Math. Soc. 39 (1964) 141-149.

20. Cobordism of combinatorial $n$-manifolds for $n \leq 8$, Proc. Camb. Phil. Soc. 60 (1964) 807-812.

21. Graded Brauer groups, J. reine angew. Math. 213 (1964) 187-199.

22. Classification problems in differential topology III. Applications to special cases, Topology 3 (1965) 291-304.

23. Quadratic forms on finite groups and related topics, Topology 2 (1963) 281-298.

24. Classification problems in differential topology VI. Classification of $(n-1)$-connected $(2 n+1)$-manifolds, Topology 6 (1967) 273-296.

25. An obstruction to finiteness of $C W$-complexes, Bull. Amer. Math. Soc. 70 (1964) 269-270.

26. Finiteness conditions for $C W$-complexes, Ann. of Math. 81 (1965) $56-89$.

27. (with W.-C. Hsiang) Orientability of manifolds for generalised homology theories, Trans. Amer. Math. Soc. 118 (1963) 352-359.

28. An extension of results of Novikov and Browder, Amer. Jour. of Math. 88 (1966) 20-32.

29. On the exactness of interlocking sequences, l'Enseignement Math. 111 (1966) 95-100.

30. Arithmetic invariants of subdivision of complexes, Canad. J. Math. 18 (1966) 92-96. 
31. Formal deformations, Proc. London Math. Soc. 16 (1966) 342-352.

32. Open 3-manifolds which are 1-connected at infinity, Quart. J. Math. Oxford 16 (1963) 263-268.

33. Survey: topology of smooth manifolds, Jour. London Math. Soc. 40 (1965) 1-20.

34. Unknotting tori in codimension one and spheres in codimension two, Proc. Camb. Phil. Soc. 61 (1965) 659-664.

35. All 3-manifolds embed in 5-space, Bull. Amer. Math. Soc. 71 (1965) 564-567.

36. Piecewise linear normal microbundles, Bull. Amer. Math. Soc. 71 (1965) 638-641.

37. Classification problems in differential topology IV. Thickenings, Topology 5 (1966) 73-94.

38. (with A. Haefliger) Piecewise linear bundles in the stable range, Topology 4 (1965) 209-214.

39. Addendum to a paper of Conner and Floyd, Proc. Camb. Phil. Soc. 62 (1966) 171-176.

40. Locally flat $P L$-submanifolds with codimension two, Proc. Camb. Phil. Soc. 63 (1967) 5-8.

41. Surgery of non simply-connected manifolds, Ann. of Math. 84 (1966) $217-276$.

42. Classification problems in differential topology V. On certain 6-manifolds, Invent. Math. 1 (1966) 355-374; corrigendum ibid. 2 (1967) 306.

43. Finiteness conditions for CW complexes II., Proc. Roy. Soc. 295A (1966) 129-139.

44. Poincaré complexes I., Ann. of Math. 86 (1967) 213-245.

45. On bundles over a sphere with fibre euclidean space, Fund. Math. 61 (1967) 57-72.

46. Homeomorphism and diffeomorphism classification of manifolds, pp. 450-460 in Proc. ICM, Moscow, 1966 Mir, 1968.

47. Graded algebras, anti-involutions, simple groups and symmetric spaces, Bull. Amer. Math. Soc. 74 (1968) 198-202.

48. Free piecewise linear involutions on spheres, Bull. Amer. Math. Soc. 74 (1968) 554-558.

49. On the axiomatic foundations of the theory of hermitian forms, Proc. Camb. Phil. Soc. 67 (1970) 243-250.

50. On groups consisting mostly of involutions, Proc. Camb. Phil. Soc. 67 (1970) 251-262.

51.a,b Geometric connectivity I., II., Jour. London Math. Soc. 3 (1971) 597-604, 605-608.

52. (with A. Fröhlich) Foundations of equivariant algebraic $K$-theory, pp. 12-27 in Lecture Notes in Math. 108, Springer (1969). 
53. On homotopy tori and the annulus theorem, Bull. London Math. Soc. 1 (1969) 95-97.

54. Non-additivity of the signature, Invent. Math. 7 (1969) 269-274.

55. (with W.-C. Hsiang) On homotopy tori II., Bull. London Math. Soc. 1 (1969) 341-342.

56. The topological space-form problem, pp. 319-351 in Topology of manifolds (ed. J.C. Cantrell \& C.H. Edwards Jr.) Markham, 1970.

57. Pairs of relative cohomological dimension 1, J. Pure Appl. Alg. 1 (1971) 141-154.

58. (with W. Browder and T. Petrie) The classification of free actions of cyclic groups of odd order on homotopy spheres, Bull. Amer. Math. Soc. 77 (1971) 455-459.

59. (with C.B. Thomas) The topological spherical space-form problem I., Compositio Math. 23 (1971) 101-114.

60. Classification of Hermitian forms I. Rings of algebraic integers, Compositio Math. 22 (1970) 425-451.

61. Geometric topology: manifolds and structures, pp. 213-219 in Proc. Internat. Cong. Math., Nice, 1970 Vol. 1 (Gauthier-Villars, 1971).

62.a Lectures on $C^{\infty}$-stability and classification, pp. 178-206

62.b Introduction to the preparation theorem, pp. 90-96

62.c Stratified sets: a survey, pp. 133-140 in Proc. Liverpool Singularities Symposium I., ed. C.T.C. Wall, Lecture Notes in Math. 192, Springer (1971).

62.d Remark on geometrical singularities, p. 121

62.e Reflections on gradient vector fields, pp. 191-195 in Proc. Liverpool Singularities Symposium II., ed. C.T.C. Wall, Lecture Notes in Math. 209, Springer (1971).

63. Classification of Hermitian forms II. Semisimple rings, Invent. Math. 18 (1972) 119-141.

64. Classification of Hermitian forms III. Complete semilocal rings, Invent. Math. 19 (1973) 59-71.

65. Classification of Hermitian forms V. Global rings, Invent. Math. 23 (1974) 261-288.

66. Quadratic forms on finite groups II., Bull. London Math. Soc. 4 (1972) 156-160.

67. (with A. Fröhlich) Equivariant Brauer groups in algebraic number theory, Bull. Math. Soc. France Mémoire 25 (1971) 91-96.

68. A remark on gradient dynamical systems, Bull. London Math. Soc. 4 (1972) 163-166.

69. On the commutator subgroups of certain unitary groups, J. Algebra 27 (1973) 306-310.

70. (with A. Fröhlich) Graded monoidal categories, Compositio Math. 28 (1974) 229-286. 
71. (with F.E.A. Johnson) Groups satisfying Poincaré duality, Ann. of Math. 96 (1972) 592-598.

72. Equivariant algebraic $K$-theory, pp. $111-118$ in New developments in topology, London Math. Soc. Lecture Notes 11 (1974).

73. Foundations of algebraic $L$-theory, pp. 266-300 in Algebraic K-theory III. Hermitian K-theory and geometric applications, ed. H. Bass, Lecture Notes in Math. 343, Springer (1973).

74. Some $L$-groups of finite groups, Bull. Amer. Math. Soc. 79 (1973) $526-529$.

75. On rationality of modular representations, Bull. London Math. Soc. 5 (1973) 199-202.

76. Periodicity in algebraic $L$-theory, pp. 57-68 in Manifolds, Tokyo 1973, Univ. of Tokyo Press, 1975.

77. Classification of Hermitian forms IV. Adèle rings, Invent. Math. 23 (1974) 241-260.

78. Norms of units in group rings, Proc. London Math. Soc. 29 (1974) 593-632.

79. Regular stratifications, pp. 332-344 in Dynamical systems - Warwick 1974, ed. A. Manning, Lecture Notes in Math. 468, Springer (1975)

80. Classification of Hermitian forms VI. Group rings, Ann. of Math. 103 (1976) 1-80.

81. Formulae for surgery obstructions, Topology 15 (1976) 189-210; corrigendum ibid 16 (1977) 495-496.

82. (with C.B. Thomas and I. Madsen) The topological spherical spaceform problem II. Existence of free actions, Topology 15 (1976) 375382.

83. Nets of conics, Math. Proc. Camb. Phil. Soc. 81 (1977) 351-364.

84. Geometric properties of generic differentiable manifolds, pp. 707-774 in Geometry and topology: III Latin American school of mathematics ed. J. Palis \& M.P. do Carmo, Lecture Notes in Math. 597, Springer (1977).

85. Free actions of finite groups on spheres, pp. 115-124 in Proc. Symp. in Pure Math. 32i (Algebraic and Geometric Topology) (ed. J. Milgram) Amer. Math. Soc. 1978.

86. Nets of quadrics and theta-characteristics of singular curves, Phil. Trans. Roy. Soc. 289A (1978) 229-269.

87. Periodic projective resolutions, Proc. London Math. Soc. 39 (1979) 509-533.

88. Note on the invariant of plane cubics, Math. Proc. Camb. Phil. Soc. 85 (1979) 403-406. 
89. (with G.P. Scott) Topological methods in group theory, pp. 137-204 in Homological group theory, ed. C.T.C. Wall and D. Johnson, London Math. Soc. Lecture Notes 36 (1979).

90. Affine cubic functions I. Functions on $\mathbb{C}^{2}$, Math. Proc. Camb. Phil. Soc. 85 (1979) 387-401.

91. Are maps finitely determined in general? Bull. London Math. Soc. 11 (1979) 151-154.

92. Singularities of nets of quadrics, Compositio Math. 42 (1981) 187212.

93. (with J.W. Bruce) On the classification of cubic surfaces, Jour. London Math. Soc. 19 (1979) 245-256.

94. Affine cubic functions II. Functions on $\mathbb{C}^{3}$ with a corank 2 critical point, Topology 19 (1980) 89-98.

95. Affine cubic functions III. Functions on $\mathbb{R}^{2}$, Math. Proc. Camb. Phil. Soc. 87 (1980) 1-14.

96. Relatively 1-dimensional complexes, Math. Zeits. 172 (1980) 77-79.

97. The first canonical stratum, Jour. London Math. Soc. 21 (1980) 419-433.

98. (omitted)

99.a A note on symmetry of singularities, Bull. London Math. Soc. 12 (1980) 169-175.

99.b A second note on symmetry of singularities, Bull. London Math. Soc. 12 (1980) 347-354.

100. Affine cubic functions IV. Functions on $\mathbb{C}^{3}$, nonsingular at infinity, Phil. Trans. Roy. Soc. 302A (1981) 415-455.

101. Stability, pencils and polytopes, Bull. London Math. Soc. 12 (1980) 401-421.

102. Finite determinacy of smooth map-germs, Bull. London Math. Soc. 13 (1981) 481-539.

103. On finite $C^{k}$ left determinacy, Invent. Math. 70 (1983) 399-405.

104. A splitting theorem for maps into $\mathbb{R}^{2}$, Math. Ann. 259 (1982) 443453.

105. Classification of unimodal isolated singularities of complete intersections, pp. 625-640 in Proc. Symp. in Pure Math. 40ii (Singularities) (ed. P. Orlik) Amer. Math. Soc., 1983.

106. Topological invariance of the Milnor number mod 2, Topology 22 (1983) 345-350.

107. (with C.B. Thomas and I. Madsen) The topological spherical spaceform problem III. Dimensional bounds and smoothing, Pacific J. Math. 106 (1983) 135-143.

108. Geometric invariant theory of linear systems, Math. Proc. Camb. Phil. Soc. 93 (1983) 57-62. 
109. Pencils of real binary cubics, Math. Proc. Camb. Phil. Soc. 93 (1983) 477-484.

110. Notes on the classification of singularities, Proc. London Math. Soc. 48 (1984) 461-513.

111. Periods of integrals and topology of algebraic varieties, Proc. Roy. Soc. 391A (1984) 231-254.

112. (with W. Ebeling) Kodaira singularities and an extension of Arnol'd's strange duality, Compositio Math. 56 (1985) 3-77.

113. (with A.A. du Plessis) On $C^{1}$-stability and $\mathcal{A}^{(1)}$-determinacy, Publ. Math. I. H. E. S., 70 (1989) 5-46.

114. Equivariant jets, Math. Ann. 272 (1985) 41-65.

115. Infinite determinacy of equivariant map-germs, Math. Ann. 272 (1985) 67-82.

116. Determination of the semi-nice dimensions, Math. Proc. Camb. Phil. Soc. 97 (1985) 79-88.

117. Survey of recent results on equivariant singularity theory, Banach Centre Publ. 20 (1988) 457-474.

118. (with J.W. Bruce and A.A. du Plessis) Determinacy and unipotency, Invent. Math. 88 (1987) 521-554.

119. (omitted)

120. Geometries and geometric structures in real dimension 4 and complex dimension 2, pp. 268-292 in Geometry and topology. Proceedings, University of Maryland 1983-1984 ed. J. Alexander and J. Harer, Lecture Notes in Math. 1167, Springer (1985).

121. Geometric structures on compact complex analytic surfaces, Topology 25 (1986) 119-153.

122. Real forms of cusp singularities, Math. Proc. Camb. Phil. Soc. 99 (1986) 213-232.

123. Real forms of smooth del Pezzo surfaces, J. reine und angew. Math. 375/376 (1987) 47-66.

124. Functions on quotient singularities, Phil. Trans. Roy. Soc. 324A (1987) 1-45.

125. Exceptional deformations of quadrilateral singularities and singular K3 surfaces, Bull. London Math. Soc. 19 (1987) 174-176.

126. Real forms of cusp singularities II., Math. Proc. Camb. Phil. Soc. 102 (1987) 193-201.

127. Deformations of real singularities, Topology 29 (1990) 441-460.

128. (with S. Edwards) Nets of quadrics and deformations of $\Sigma^{3(3)}$ singularities, Math. Proc. Camb. Phil. Soc. 105 (1989) 109-115.

129. Elliptic complete intersection singularities, pp. 340-372 in Singularity theory and its applications. Warwick, 1989, I., ed. D. Mond and J. Montaldi, Lecture Notes in Math. 1462, Springer (1991). 
130. Pencils of cubics and rational elliptic surfaces, pp. 373-405 in Singularity theory and its applications. Warwick, 1989, I., ed. D. Mond and J. Montaldi, Lecture Notes in Math. 1462, Springer (1991).

131. Root systems, subsystems and singularities, Jour. Alg. Geom. 1 (1992) 597-638.

132. Is every quartic a conic of conics? Math. Proc. Camb. Phil. Soc. 109 (1991) 419-424.

133. (with A.A. du Plessis) Topological stability, pp. 351-362 in Singularities, Lille 1991, ed. J.-P. Brasselet, London Math. Soc. Lecture Notes 201, Cambridge University Press, 1994.

134. Weighted homogeneous complete intersections, pp. 277-300 in Algebraic geometry and singularities (proceedings of conference at La Rabida 1991), ed. A. Campillo López and L. Narváez Macarro, Progress in Math. 134, Birkhäuser, 1996.

135. Classification and stability of singularities of smooth maps, pp. 920952 in Singularity Theory (Proceedings of College on Singularities at Trieste 1991), ed. D.T. Lê, K. Saito and B. Teissier, World Scientific, 1995.

136. Duality of singular plane curves, Jour. London Math. Soc., 50 (1994) 265-275.

137. Quartic curves in characteristic 2, Math. Proc. Camb. Phil. Soc. 117 (1995) 393-414.

138. Geometry of quartic curves, Math. Proc. Camb. Phil. Soc., 117 (1995) 415-423.

139. Highly singular quintic curves, Math. Proc. Camb. Phil. Soc., 119 (1996) 257-277.

140. Duality of real projective plane curves: Klein's equation, Topology, 35 (1996) 355-362.

141. Real rational quartic curves, pp. 1-32 in Real and complex singularities, ed. W.L. Marar, Pitman Research Notes in Math. 333, Longman, 1995.

142. Pencils of binary quartics, Rend. Sem. Mat. Univ. Padova 99 (1998) 197-217.

143. (with A.A. du Plessis) Discriminants and vector fields, pp. 119-140 in Singularities (Oberwolfach 1996), Progr. Math. 162, Birkhäuser, 1998.

144. (with A.A. du Plessis) Versal deformations in spaces of polynomials with fixed weight, Compositio Math. 114 (1998) 113-124.

145. (with A.A. du Plessis) Applications of the theory of the discriminant to highly singular plane curves, Math. Proc. Camb. Phil. Soc. 126 (1999) 259-266.

146. Newton polytopes and non-degeneracy, J. reine angew. Math. 509 (1999) 1-19. 